\title{
RELACIÓN ENTRE CARIES DENTAL Y FACTORES DE RIESGO EN NIÑOS DE HOGARES INFANTILES DEL BIENESTAR FAMILIAR DE CARTAGENA
}

\author{
${ }^{1}$ Farith González Martínez, ${ }^{2}$ Luz Maida Luna Ricardo, ${ }^{3}$ María Yolanda Solana \\ ${ }^{1}$ Odontólogo U. de Cartagena, Especialista en Investigación Social U. de Cartagena, \\ Magíster en Salud Pública Universidad Nacional de Colombia. \\ ${ }^{2}$ Odontóloga U. de Cartagena, Odontopediatra Especialista en Investigación Social U. de Cartagena. \\ ${ }^{3}$ Especialista en Odontopediatría, U. de Cartagena.
}

Autor responsable de correspondencia: Farith González Martínez.

Correo electrónico: farithgm@hotmail.com

\section{RESUMEN}

Objetivo: Describir la ocurrencia de la caries en niños de hogares infantiles del (ICBF) Cartagena y relacionarlo con los factores de riesgo para la caries dental.

Materiales y Métodos: Estudio de corte transversal, cuya muestra fueron 95 niños entre 5 y 6 años seleccionados en forma aleatoria de cinco escuelas del ICBF de Cartagena. Las variables fueron: presencia de caries, experiencia de caries, dieta cariogénica, índice de placa bacteriana mayor del 15\%, responsabilidad del cepillado delgada en el niño, frecuencia de cepillado de una vez/día, no exposición a fluoruros, hijos de padres separados, escolaridad de los padres inferior a secundaria, niños que asisten a consulta odontológica menos de una vez/año, niños que no realizan actividades recreativas, niños con mala conducta escolar, edad y género.

Resultados: Se encontró una prevalencia de caries del 51\%, los factores de riesgo de mayor frecuencia fueron: la placa $>15 \%$ con el $75 \%$, ninguna práctica de actividades recreativas con 69\% y la experiencia de caries el 58\%. Con respecto a las razones de disparidad (OR), se encontró asociación estadísticamente significativa entre la ocurrencia de caries y la experiencia de caries $\mathrm{OR}=18.3$, la escolaridad de los padres inferior a secundaria $\mathrm{OR}=11.1$, la delegación de la responsabilidad del cepillado en el niño, $\mathrm{OR}=3.25$ y la ninguna práctica de actividades recreativas, $\mathrm{OR}=2.57$.

Conclusiones: El comportamiento de la caries en esta población estuvo influido por estas cuatro variables, las cuales se pueden conjugar en diferentes circunstancias para producir las diferencias a nivel individual. [González F, Luna LM, Solana MY. Relación entre caries dental y factores de riesgo en niños de hogares infantiles Bienestar Familiar de Cartagena. Ustasalud 2008; 7: 87 - 95]

Palabras clave: Prevalencia de caries, Riesgo biológico, Riesgo social, Niños preescolares.

\section{RELATIONSHIP BETWEEN DENTAL CARIES AND RISK FACTORS AMONG CHILDREN OF FIVE FAMILY WELFARE HOUSES IN THE CITY OF CARTAGENA}

\begin{abstract}
Objective: To describe the occurrence of caries in children of the Colombian Family Welfare Institute houses in Cartagena and the relation with risk factors for dental caries.

Material and Methods: This study is cross-sectional. The sample was composed of 95 children between 5 and 6 years of age selected randomly of 5 schools of the Cartagena Family Welfare Institute. The variables were: presence of caries, caries experience, cariogenic diet, bacterial plaque $>15 \%$, responsibility of brushing thin on the child, frequency of brushing once per day, no exposition to fluorides, children of separated parents, parents of school less than secondary children attending dental consultation less than once per year, children who do not perform recreational activities, school children with misconduct, age and gender.

Results: Caries prevalence was found with $51 \%$, the risk factors with greater prevalence were: plaque $>$ than $15 \%$ with $75 \%$, no recreation activity practices $69 \%$, experience with caries $58 \%$. In reference with the disparity reasons (DR), there was found a statistically significant association between the occurrence of caries and the experience of caries DR=18.3; with parent's low educational level $\mathrm{DR}=11.1$; with the responsibility on children for their own tooth brushing $\mathrm{DR}=3.25$; and no recreation activity practice $\mathrm{DR}=2.57$. Conclusions: The behavior of dental caries in this population was influenced by these four variables, which can be combined in different circumstances to produce the differences at a individual level.
\end{abstract}

Key words: Caries prevalence, Biological risk, Social risk, Preschool children

Recibido para publicación: 27 de marzo de 2008. Aceptado para publicación: 2 de febrero de 2009. 


\section{INTRODUCCIÓN}

La caries dental se considera un problema de salud pública en nuestro país, al analizar los datos del III Estudio Nacional de Salud Bucal (ENSAB), ${ }^{1}$ en edades de 5 años, la prevalencia fue del $54.8 \%$ y el $60.4 \%$ de los niños tuvo historia de la enfermedad. En la Costa Atlántica incluido Cartagena, los resultados son similares, reportándose una prevalencia del 55.6\% y una historia de caries del $60.1 \%$. Aunque estos resultados pueden representar el impacto de las acciones educativas en dentición temporal que se han desarrollado en el país en los últimos 20 años, todavía no hay datos nacionales que logren poner a prueba asociaciones entre los factores de riesgo y la ocurrencia de la enfermedad.

En este orden de ideas, es importante resaltar que en la ciudad de Cartagena existen poblaciones con diversidad de exposiciones a los factores de riesgo para Caries, por ello se generó esta propuesta, ya que, a pesar de que hay suficiente evidencia sobre el origen de la caries y sus factores condicionantes, el estudio ha sido realizado en otras poblaciones, que tienen costumbres y estilos de vida diferentes, que pueden representar discrepancias al momento de utilizarlos para valorar el riesgo individual en la población objeto de este estudio., ${ }^{2,3}$

Este estudio se justifica debido a que con los resultados se podrían diseñar mejores y más acertadas herramientas preventivas que lograrían aproximarnos en la búsqueda de un modelo predictor de caries para niños con estas mismas características, obteniéndose desde la academia la credibilidad y el liderazgo que se ha perdido a nivel comunitario.

Actualmente, los factores considerados en la literatura como de riesgo que pueden contribuir a la presencia de caries dental, tienen que ver con la parte biológica (historia pasada de caries, placa bacteriana, estado de erupción, macromorfología, saliva, dieta, exposición a fluoruros, incapacidad fisica), y por otro lado el componente social (perfil familiar, nivel sociocultural, estrato socioeconómico y estilo de vida). ${ }^{4,5}$

En lo que tiene que ver con los factores biológicos, se considera de mayor utilidad de acuerdo a nuestro entorno; la historia pasada de caries a través del índice coe, el estado de higiene oral a través de la valoración del índice de placa bacteriana, la dieta a través de sus efectos locales y sistémicos, la saliva a través de la valoración de los efectos de los medicamentos cuando se padece de enfermedades sistémicas y la exposición de fluoruros; desde lo sistémico a partir de los alimentos y lo local en la aplicación de topicaciones. ${ }^{6-13}$
Uno de los principales factores biológicos utilizados durante mucho tiempo a nivel comunitario ha sido la historia pasada de caries, que ayuda a sospechar si un individuo ha estado expuesto o no a factores que han permitido o evitado el desarrollo de la enfermedad. A pesar de lo anterior, los índices de caries han sido criticados, debido a que no permiten evidenciar con certeza si un diente se ha obturado o perdido por caries o por otras razones. En este sentido, Steiner y colaboradores (1992), estudiaron algunos predictores para el incremento de caries en una población infantil, ${ }^{14} \mathrm{y}$ resaltaron que los mejores predictores eran: un bajo número de temporales sanos y un alto número de lesiones precavitacionales en primeros molares permanentes, de esta manera, se puede recobrar el valor de la historia de caries como un factor de riesgo más. Por otra parte, los mismos autores encontraron, que el índice $\mathrm{COP} /$ ceo es inferior para predecir el incremento de caries. Sin embargo, en un estudio realizado en el país en adolescentes entre 15 y 19 años, por López y Cerezo se encontró que la concordancia para la predicción de caries con respecto a la historia pasada de caries fue buena, con una sensibilidad del $99.39 \% .{ }^{15}$ Esta evidencia puede aportar elementos de juicio para poder evaluar este factor como de riesgo para la caries dental en niños con dentición temporal.

Por otro lado, en lo concerniente a los factores sociales, éstos están enmarcados dentro del núcleo familiar, ya que es la institución de mayor importancia en lo educativo desde el primer momento en que el niño nace. En este sentido, se supone que los padres son los encargados de evitar que los niños se expongan a los factores de riesgo que inciden en la enfermedad oral, constituyéndose en los primeros maestros durante los inicios de la vida. Al igual que la familia, la escuela se constituye en otro escenario propicio para fomentar una educación que incluya hábitos y conductas saludables en forma permanente, de esta manera, son los maestros los responsables del mantenimiento de estas, monitoreando todos los aspectos que pueden influir en los cambios en el estilo de vida. De esta forma, las madres son el principal transmisor de los hábitos de salud y las creadoras de patrones de comportamientos para los niños. ${ }^{13}$ Por otra parte, el estrato socioeconómico puede influir en estos malos hábitos. En la actualidad se ha descrito una relación directa entre estrato y salud, debido a que un individuo de bajos recursos tiene menos posibilidades de tener acceso a una buena alimentación, vivienda digna, una buena educación y la compra de elementos de higiene oral útiles para evitar enfermar. ${ }^{16-19}$

Adicionalmente a los factores descritos, el empleo y estatus ocupacional representan un estilo de vida 
y con frecuencia determinan la facilidad o el acceso hacia algún tipo de servicios de salud, por lo tanto, las visitas al odontólogo están estrechamente relacionadas con el ingreso familiar. ${ }^{20-21}$ Actualmente, existe una relación significativa entre el nivel educativo de los padres y el desarrollo de la enfermedad, debido a que el conocimiento de los padres sobre las medidas preventivas a nivel oral influye en que éstas sean utilizadas en el hogar y de alguna manera causen algún efecto sobre la ocurrencia de la enfermedad. $22-24$

El objetivo general fue describir la ocurrencia de la caries dental en niños de hogares infantiles del Instituto Colombiano de Bienestar Familiar (ICBF) de Cartagena y relacionarlo con los factores que reporta la literatura como de riesgo para caries dental.

\section{MATERIALES Y MÉTODOS}

Estudio de corte transversal cuya población estudiada fueron los niños entre 5 y 6 años de cinco escuelas comunitarias del ICBF de Cartagena. Se seleccionó una muestra representativa de 95 niños, 61 de cinco años y 34 de seis años. En cuanto el género, 60 hombres y 35 mujeres. En total, fueron evaluadas 9700 superficies en 1940 dientes. La selección de los niños fue realizada a través de un muestro aleatorio simple, se tomó como población de referencia la totalidad de los niños de las cinco escuelas objeto de estudio. Como criterios de selección se tuvieron en cuenta que fueran nacidos y residentes en Cartagena con dirección confirmada, sin ningún compromiso motriz o sistémico y consentimiento informado por parte de los padres.

Las variables se clasificaron en: variables de salida: (prevalencia de experiencia de caries) y variables explicatorias: experiencia de caries (coes), dieta cariogénica, índice de placa bacteriana mayor del $15 \%$, responsabilidad del cepillado delegada en el niño, frecuencia de cepillado de una vez/día, no exposición a fluoruros, hijos de padres separados, escolaridad de los padres inferior a secundaria, niños que asisten a consulta odontológica menos de una vez/año, niños que no realizan actividades recreativas y niños con mala conducta escolar.

Prevalencia de experiencia de caries: Se valoró la presencia de niños con coe-s mayor o igual a 1 .

Ceo-s: se evaluó el promedio de lesiones cavitacionales, precavitacionales y/o microcavitacionales, así como los dientes con superficies obturadas y con extracción indicada. (ICDAS Modificado)

Experiencia de caries: Se evaluó el promedio de superficies con coe-s
Dieta cariogénica: El tipo de dieta se evaluó a través de encuestas. En primera instancia se valoró el número de veces al día que el niño consume carbohidratos y azúcares, se tuvo como riesgo el consumo de más de 3 momentos diarios de carbohidratos y/o dulces o bebidas azucaradas. (Criterios Encuesta Nacional de la Situación Nutricional ENSIN 2005)

Placa bacteriana: Se evaluó como riesgo clínicamente la presencia de placa mayor del $15 \%$ a través de un control de placa bacteriana madura (Índice de Placa Comunitario).

Exposición a fluoruros: A través de la encuesta realizada a los padres se determinó como riesgo la ninguna asistencia a campañas de fluorización o aplicación de flúor a nivel local en el consultorio.

Frecuencia de cepillado: A través de encuesta se evaluó como riesgo los niños que se cepillaban sólo una vez al día.

Responsabilidad del cepillado delegada en el niño: A través de encuesta se valoró como riesgo los niños que se cepillaban solos sin ninguna supervisión de un adulto.

Estado civil de los padres: A través de encuestas se incluyó como riesgo a los niños que vivían en hogares con padres separados o fallecidos.

Nivel escolaridad de los padres: A través de encuestas se valoró como riesgo a los padres que no hubiesen terminado la secundaria. Por otro lado, se evaluó la información sobre higiene oral que habían recibido los padres.

Estilo de vida de los niños: A través de encuestas se valoró como riesgo si el niño no realizaba ninguna actividad recreativa. Además se evaluó la mala conducta escolar del niño.

Consulta odontológica al año: A través de encuestas se indagó como riesgo la no asistencia a consulta odontológica al menos una vez al año.

Variables sociodemográficas: edad y sexo, distribuidas a nivel dicotómico en 5 y 6 años y masculino y femenino respectivamente.

Cuestionario: En cuanto a los procedimientos y técnicas utilizadas para la recolección de datos, en primera instancia se realizó el diseño una encuesta estructurada dirigida a los padres de los menores para la medición de los factores asociados, la cual fue valorada inicialmente por dos expertos con amplia experiencia en la construcción de estos instrumentos, se busca obtener validez de apariencia y de contenido, reevaluando las preguntas que no midieran en forma objetiva las variables de estudio, luego de esto se estandarizaron a los encuestadores 
y anotadores. Por otro lado, para la valoración de la dieta, se utilizaron los criterios diagnósticos del ICBF aplicados en la ENSIN 2005. ${ }^{25}$

Para la recolección de la información propiamente dicha se convocó a los padres que fueron seleccionados durante el muestreo y, por ser un estudio con mediciones sobre seres humanos, se requirió el uso de un consentimiento informado, para ser diligenciado por los padres o personas responsables de los niños, en donde se les explicó el propósito del estudio y los procedimientos, de acuerdo a la resolución 8430 de 1993 del Ministerio de Salud de la república de Colombia, indicándose que estas actividades diagnósticas generarían un riesgo mínimo para los sujetos evaluados y se ratificó el compromiso de los investigadores para solucionar los inconvenientes que surgieran de este proceso. ${ }^{26}$

Evaluación clínica: Las variables consideradas evento (caries) y el factor de riesgo placa bacteriana fueron medidas a través de un instrumento clínico. (Para la caries se diseñó un formato basado en los criterios reportados en la Guía de Práctica clínica en salud oral de la Secretaría de salud Bogotá ACFO 2007) y para la Placa Bacteriana se utilizó el índice de Placa comunitario de Corchuelo 1996. ${ }^{27}$
El procedimiento se inició con la medición de la higiene oral a través del índice de placa bacteriana, el cual consistió en la aplicación de sustancia reveladora ditono a nivel de todas las superficies dentales, exceptuadas las caras palatinas de los incisivos superiores y las superficies interproximales de todos los dientes, las cuales fueron manchadas por un operador con el fin de que la tinción fuera uniforme, luego se realizó la observación del número de superficies manchadas solamente de color violeta y se dividió por el número de superficies evaluadas.

Posteriormente, para realizar el diagnóstico de caries dental se utilizaron criterios de caries (ICDAS Modificado), ${ }^{28}$ manejados por dos examinadores calibrados a través de un estándar de oro, con valores Kappa interexaminador de 0.80 e intraexaminador de 0.85 . De esta forma se obtuvo la presencia de caries y la prevalencia de experiencia de caries. Inicialmente, se realizó la profilaxis con instrumento rotatorio de baja velocidad y piedra pómez con glicerina, para la eliminación de todas las pigmentaciones asociadas con placa y permitir la visibilidad requerida para observar los cambios de color u opacidades dentarias, con los siguientes códigos y criterios.

\begin{tabular}{|c|l|}
\hline Código & \multicolumn{1}{|c|}{ ICDAS Modificado } \\
\hline $\mathbf{0}$ & Ningún cambio en la translucidez del esmalte después de secado prolongado con aire \\
\hline $\mathbf{1 b}$ & Opacidad café cariosa visiblemente distinguible confinada a la fisura \\
\hline $\mathbf{2 b}$ & Opacidad café cariosa visiblemente distinguible más allá de la fisura \\
\hline $\mathbf{1 w}$ & Opacidad blanca cariosa distinguible sólo después de secado prolongado con aire \\
\hline $\mathbf{2} \mathbf{w}$ & Opacidad blanca cariosa visiblemente distinguible en superficie húmeda \\
\hline $\mathbf{3}$ & Después de secar se observa una pérdida cariosa de integridad superficial sin exposición de dentina \\
\hline $\mathbf{4}$ & Sombra de dentina decolorada visible a través de una superficie de esmalte aparentemente intacta \\
\hline $\mathbf{5}$ & Cavidad en esmalte opaco o decolorado con base expuesta en dentina \\
\hline $\mathbf{6}$ & Cavidad extensa profunda o amplia y la dentina es visible tanto en las paredes como en la base \\
\hline
\end{tabular}

Fuente: ICDAS: Ismail A. Detroit Dental Health Project, 2002. - ICDAS: Ekstrand K. Assessment of activity of caries, 2003.

Para evaluar las obturaciones se utilizaron los criterios de adaptación compatible con salud, si no se observó ningún cambio de opacidad en el tejido dentario adyacente a la obturación, se incluyó en los datos corres- pondientes a obturaciones. Por último, se evaluaron los dientes con extracción indicada, los cuales fueron incluidos solo aquellos que se habían perdido por caries, según la historia de la enfermedad. 
Análisis estadístico: En cuanto al análisis de los datos, inicialmente se utilizaron pruebas de estadística descriptiva, posteriormente se estimó la ocurrencia de la caries y de los factores de riesgo a través de prevalencias, calculándose los estimadores mediante el programa estadístico STATA para Windows 9,0. Luego se pusieron a prueba asociaciones por medio de razones de disparidad (OR), con intervalos de confianza del 95\%. Por último, para establecer la significación del estimador de asociación se aplicó la prueba Chi- cuadrado, se reconoció como significativos valores de probabilidad menores a 0.05 .

\section{RESULTADOS}

La prevalencia de experiencia de caries fue del 51\% (IC: 95\%; 40\% - 60\%). El promedio del coe-s fue de $2.66(\mathrm{DE}=3.4)$, siendo más frecuentes las lesiones cariosas con 2.31 (IC: 95\%; $1.55-3.17, \mathrm{DE}=2.13$ ). Según los criterios diagnósticos ICDAS Modificado, las lesiones precavitacionales (1b, $2 \mathrm{~b}, 1 \mathrm{w}, 2 \mathrm{w}$ ) se presentaron con el mayor promedio 1.66 (IC: $95 \%$; $0.87-2.39, \mathrm{DE}=$ 1.03) seguido de las cavitacionales $(5,6)$ con 0.39 (IC: $95 \% ; 0.33-0.45, \mathrm{DE}=0.3$ ). Al relacionar estos valores con el género, el masculino obtuvo mayor ocurrencia con 53\% (IC: 95\%; 41\% - 66\%) y en cuanto a la edad no se encontraron diferencias estadísticamente significativas (Tablas 1 y 2).

En cuanto a la prevalencia de los factores asociados a caries, los de mayor ocurrencia fueron la placa $>15 \%$ con el 75\%, (IC: 95\%; 66\%- 84\%), ninguna práctica de actividades recreativas con 69\% (IC: 95\%; 60\% - 79\%), la experiencia de caries con $58 \%$, (IC: $95 \%$; $48 \%$ - 68\%), la ninguna exposición a fluorizaciones con 52\%, (IC: 95\%; 41\% - 62\%), la responsabilidad del cepillado delegada en el niño con el 50\%, (IC: 95\%; $43 \%-57 \%$ ) y la escolaridad de los padres inferior a secundaria con 48\%, (IC: 95\%; 38\% - 58\%) (Tabla 3).

Con respecto al análisis bivariado, se encontró asociación estadísticamente significativa entre la ocurrencia de caries y la experiencia de caries, $\mathrm{OR}=$ 18.3 (IC: 95\%; 6.29 - 53.2, P<0.000), la escolaridad de los padres inferior a secundaria con $\mathrm{OR}=11.1$ (IC:95\%; 4.26 - 28.8, P<0.000), la delegación de la responsabilidad del cepillado en el niño, $\mathrm{OR}=3.25$ (IC:95\%;1.40 - 7.56, $\mathrm{P}=0.006$ ) y la ninguna práctica de actividades recreativas, $\mathrm{OR}=2.57$ (IC:95\%;1.03 6.39, $\mathrm{P}=0.03$ ) (Tabla 4 y 5).

Tabla 1. Ocurrencia y promedio de caries con respecto a variables sociodemográficas.

\begin{tabular}{|c|c|c|c|c|c|}
\hline & Número & Prevalencia (\%) & IC: $95 \%$ & coe-s & $\mathrm{DE}$ \\
\hline \multicolumn{6}{|l|}{ Género } \\
\hline Masculino & 32 & 53 & $41-66$ & 2.5 & 3.55 \\
\hline Femenino & 16 & 46 & $29-62$ & 2.94 & 3.28 \\
\hline \multicolumn{6}{|l|}{ Edad } \\
\hline 5 & 31 & 51 & $38-63$ & 2.57 & 3.76 \\
\hline 6 & 17 & 50 & $33-66$ & 2.82 & 2.82 \\
\hline Total & 48 & 51 & $40-60$ & 2.66 & 3.4 \\
\hline Tipo de lesión & & & & IC: $95 \%$ & DE \\
\hline Precavitacional (1b, 2b, 1w, 2w) & & & & $0.87-2.39$ & 1.03 \\
\hline Microcavitacional, sombra gris $(3,4)$ & & & & $0.13-0.43$ & 0.74 \\
\hline Cavitacional $(5,6)$ & & & & $0.33-0.45$ & 0.3 \\
\hline Total & & & & $1.55-3.17$ & 2.13 \\
\hline
\end{tabular}


Tabla 3. Ocurrencia de los factores asociados a caries dental.

\begin{tabular}{lccc}
\hline Factores asociados & Número & Ocurrencia (\%) & IC: 95\% \\
\hline Experiencia de caries & 162 & 58 & $48-68$ \\
Placa bacteriana mayor del 15\% & 143 & 75 & $66-84$ \\
Dieta cariogénica & 133 & 40 & $30-50$ \\
Responsabilidad del cepillado en niño & 114 & 50 & $43-57$ \\
Frecuencia de cepillado 1 vez día & 48 & 46 & $36-46$ \\
Consulta odontológica menor 1 vez/año & 114 & 19 & $11-27$ \\
Ninguna exposición a flúor & 92 & 52 & $41-62$ \\
Escolaridad padres inferior a secundaria & 154 & 48 & $38-58$ \\
Ninguna información higiene oral padres & 109 & 36 & $26-46$ \\
Niños con padres separados/madre soltera & 78 & 38 & $28-48$ \\
Ninguna actividad recreativa & 32 & 69 & $60-79$ \\
Mala conducta escolar del niño & 46 & 35 & $25-44$ \\
\hline
\end{tabular}

Tabla 4. Ocurrencia de caries asociado con los indicadores biológicos.

\begin{tabular}{lccccc}
\hline \multicolumn{1}{c}{ Variables } & Caries & No caries & OR & IC: $95 \%$ & Valor P \\
\hline Índice de PB. >15\% & 38 & 33 & 1.61 & $0.63-4.11$ & 0.31 \\
Experiencia de caries & 42 & 13 & 18.3 & $6.29-53.2$ & $<0.0001^{*}$ \\
Dieta cariogénica & 19 & 19 & 0.96 & $0.42-2.19$ & 0.93 \\
Ninguna exposición a flúor & 26 & 23 & 1.23 & $0.55-2.76$ & 0.61 \\
Cepillado 1 vez/día & 19 & 20 & 0.88 & $0.39-2.0$ & 0.76 \\
Niño responsable del cepillado & 29 & 15 & 3.25 & $1.40-7.56$ & $0.006^{*}$ \\
\hline
\end{tabular}

* Asociación estadísticamente significativa.

Tabla 5. Ocurrencia de caries asociado con los indicadores sociales.

\begin{tabular}{lccccc}
\hline \multicolumn{1}{c}{ Variables } & Caries & $\begin{array}{c}\text { No } \\
\text { caries }\end{array}$ & OR & IC: $95 \%$ & Valor P \\
\hline Escolaridad de padres Inferior a secundaria & 36 & 10 & 11.1 & $4.26-28.8$ & $<0.0001^{*}$ \\
Padres con poca información sobre higiene oral & 19 & 15 & 1.39 & $0.60-3.24$ & 0.43 \\
Niños con Padres separados & 20 & 16 & 1.38 & $0.60-3.18$ & 0.44 \\
Ninguna actividad recreativa & 38 & 28 & 2.57 & $1.03-6.39$ & $0.03^{*}$ \\
Ninguna consulta odontológica al año & 6 & 12 & 0.41 & $0.14-1.42$ & 0.11 \\
Mala conducta escolar del niño & 19 & 14 & 1.54 & $0.65-3.61$ & 0.31 \\
\hline
\end{tabular}

* Asociación estadísticamente significativa. 


\section{DISCUSIÓN}

La ocurrencia de caries observada en esta población se considera alta, por tal motivo, los resultados obtenidos pueden ser útiles para tomar decisiones de salud pública al momento de plantear estrategias preventivas en poblaciones de alto riesgo.

Al comparar los resultados obtenidos de experiencia de caries, éstos son menores a los reportados en otras regiones del país. Saldarriaga y colaboradores, ${ }^{3}$ en niños entre 3 y 5 años del ICBF de Medellín, a través de los criterios diagnósticos modernos (Pitts y Fyffe) encontraron valores de coe-s en 5.5 y en cuanto a la ocurrencia de caries, obtuvieron un $83.7 \%$. Estas diferencias pueden explicarse debido a que en este último estudio las edades fueron menores y los primeros dientes temporales todavía no habían exfoliado, lo que permite un incremento en el coe. Además, los niños de hogares infantiles del ICBF de Cartagena estuvieron inmersos dentro de un programa de prevención de caries en el 2003. ${ }^{29}$ En este mismo sentido, en un estudio realizado por González y colaboradores en niños de hogares infantiles de Bogotá reportó un ceo de 3.3 y una prevalencia de experiencia de caries del $70 \%{ }^{30}$ Por otro lado, al confrontar estos índices a nivel local, en poblaciones vulnerables sin ninguna intervención, las diferencias se acentuaron; en un estudio realizado por González y colaboradores en niños escolares desplazados por la violencia en Cartagena de Indias, ${ }^{4}$ se observó un ceo-s de 16.1, y otro estudio realizado en niños escolares de la BoquillaCartagena por González y colaboradores se reportó un ceo-s de 18.0. ${ }^{6}$

En la puesta a prueba de asociaciones entre caries y los factores asociados, en este estudio sólo se encontraron estimadores con fuerza estadística con la prevalencia de experiencia de caries, la ninguna práctica de actividades recreativas, la escolaridad de los padres inferior a secundaria y la responsabilidad del cepillado delegada en el niño. En este sentido, en el país hay pocos estudios que demuestren validez o que sean comparables, no obstante, hay reportes con resultados similares por Rey y colaboradores, ${ }^{7}$ en niños escolares de Moniquirá Boyacá, los cuales encontraron que sólo la experiencia de caries mostró una asociación estadísticamente significativa con un OR de 4.8. En este orden de ideas, la experiencia de caries ha sido el factor de riesgo de mayor fuerza estadística debido probablemente a que una lesión cavitacional ofrece un ambiente favorable para que se retenga la placa bacteriana.

En cuanto al papel de los padres en mantener los buenos hábitos higiénicos orales, autores como Cuartas y colaboradores, ${ }^{8}$ en niños de hogares infantiles de Envigado, Antioquia encontraron dife- rencias estadísticamente significativas entre la percepción de los hábitos de higiene oral de los padres y el estado de salud oral de los niños. Por otra parte, algunos investigadores han confrontado los conocimientos y prácticas de salud oral de sus padres con respecto a la historia de la enfermedad de sus hijos, tal es el caso de Franco y colaboradores, ${ }^{9}$ quienes en niños menores de 6 años de la ciudad de Medellín, encontraron una diferencia para la prevalencia de caries, especialmente para el estrato medio alto con un $48.4 \%$ y para el bajo con el $58.3 \%$. Además hubo diferencias en el nivel de escolaridad de las madres, ya que el $42 \%$ de las madres del estrato medio alto y el $50 \%$ del bajo afirman no haber recibido información sobre el cuidado de salud oral de sus hijos. En este mismo sentido, Navas y colaboradores encontraron una relación estadísticamente significativa entre la educación de los padres y la caries de sus hijos, y reportaron que los hijos de padres que no han tenido un nivel educativo favorable presentan un alto índice de caries..$^{10}$ Por otro lado, en niños de 6 años en Brasil, Pérez y colaboradores encontraron un OR de 2.2 en padres con menos de 8 años de estudio cuando el niño nació. ${ }^{31}$ Esto coincide con los resultados de este estudio con respecto al mayor riesgo de caries que tienen los niños de padres con bajo nivel de escolaridad (secundaria incompleta) lo que los lleva a no influir en la salud oral de sus hijos por los pocos conocimientos que se tienen en la prevención de esta enfermedad, al dejar en el niño la responsabilidad de su cepillado. Por otro lado, con respecto a la responsabilidad del cepillado se han reportado estudios en México por Cook y colaboradores quienes encontraron diferencias estadísticamente significativas en la severidad de la caries en niños entre 1 y 5 que eran cepillados por sus padres con respecto a los que se cepillaban solos, ${ }^{32}$ estos resultados coinciden con nuestro estudio probablemente por que el niño en estas edades no ha adquirido la motricidad fina adecuada para realizar un buen cepillado y por otro lado, sus conocimientos en higiene oral son muy escasos sin una conciencia plena para ponerlos en práctica.

Por último con respecto a la ocurrencia de caries y estilo de vida, Blinkhorn encontró coincidencias al relacionar la ninguna práctica de deportes o actividades recreativas y la presencia de caries, ${ }^{11}$ ya que los niños que se mantenían ocupados con algunos juegos de carácter físico o mental presentaron menos dientes cariados en relación a los que no hacían deporte. En nuestro estudio se reafirma esta evidencia y aunque a pesar de que la muestra estuvo constituida por niños preescolares (5 y 6 años), ya se motivan a participar en actividades recreativas o culturales dentro de la escuela o en el hogar, lo 
que puede contribuir a que tengan disciplina en los horarios destinados para la higiene oral.

Se puede concluir que la ocurrencia de caries en esta población puede estar relacionada con éstos cuatro factores asociados (experiencia de caries, ninguna práctica de actividades recreativas, escolaridad de los padres inferior a secundaria y la responsabilidad del cepillado delegada en el niño), evidenciándose que el comportamiento de esta enfermedad está influido por múltiples variables, las cuales se pueden conjugar en diferentes circunstancias para producir las diferencias a nivel individual.

\section{Agradecimientos}

A todos los hogares del Instituto de Bienestar Familiar involucrados en este estudio, a los padres de los niños y a la universidad de Cartagena, postgrado de Odontopediatría por todo el apoyo académico y administrativo brindado a los investigadores.

\section{BIBLIOGRAFÍA}

1. Ministerio de Salud - República de Colombia, Centro Nacional de Consultoría CNS: III- ENSAB III; Tomo VII. Bogotá: Lito Servicios ALER; 1999; $84-88$.

2. Haunsen H, Seppa L, Fejerskov O. "Can Caries by Predicted" cap. 20. Textbook of Clinical Cariology. 2nd. Ed. Munksgaard. Copenhaguen 1994; 84 - 85.

3. Saldarriaga A, Franco A, González S, Escobar A, Cordero N, Dávila H. Evaluación del subregistro de caries dental en dentición decidua. CES Odontología 2003; 6: 43 - 47.

4. González F, Carmona L, Pérez G. Prevalencia de caries dental "Ekstrand" y factores de riesgo en niños escolarizados con edades entre 5 y 13 años del barrio Nelson Mandela de la ciudad de Cartagena de Indias. Memorias XVI Encuentro Nacional de Investigación Odontológica Universidad El Bosque- ACFO Bogotá 2004; 35.

5. Martignon S, González M, McCornikc V, Ruiz JA, Jácome S, Guarnizo C. Guía de Diagnóstico, Prevención y Tratamiento de la Caries Dental. Asociación Colombiana de Facultades de Odontología - ACFO., Secretaría Distrital de Salud Bogotá. Editor: Secretaría Distrital de Salud Bogotá, Bogotá, 2007; 79 - 82.

6. González F, Alfaro L, Nieto C, Carmona L. Evaluación de las condiciones de salud oral y los conocimientos en niños escolares entre 7 y 14 años de la población de la BoquillaCartagena de Indias 2005. Revista Científica Facultad de Odontología Universidad El Bosque 2006; 12: 25 - 34.

7. Rey M, Salas E, Martignon S. Caries dental y factores de riesgo asociados en la población escolar de Moniquirá, Boyacá. Revista Científica Facultad de Odontología Universidad El Bosque 2003; 9: 28 - 34.

8. Cuarta J, Álvarez A, Ospina A, Maya A, Cárdenas J, Arias M, Jaramillo A. Relación entre la percepción de los padres sobre el tratamiento odontológico y sus hábitos de higiene oral, con la historia de caries dental en sus hijos, entre 3 y 5 años de edad. CES Odontología 2002; 15: 13 - 18.

9. Franco A, Santamaría A, Kurzer E, Castro L, Giraldo M. El menor de seis años: Situación de caries y conocimientos y prácticas de cuidado bucal de sus madres. CES Odontología 2004; 17: 19 - 29.
10. Navas R, Rojas T. Salud Bucal en preescolares; su relación con factores sociales. Memorias XIV Encuentro Nacional y III Latinoamericano de Investigación Odontológica. ACFO- Medellín 2004; 23.

11. Blinkhorn AS. Factors affecting the compliance of patients with preventive dental regimens. Int Dent J 1993; 43: 294 - 298.

12. González F, Luna L, Martínez N, Solana M. Correlación entre los factores de riesgo biológicos y sociales con la presencia de caries dental en niños entre 5 y 6 años de hogares infantiles del Instituto Colombiano de Bienestar familiar de la Cuidad de Cartagena, 2005-2006. Revista de la Federación Odontológica Colombiana 2007; 69: 7- 20.

13. Duque J, Rodríguez A, Coutin G, Riveron F. Factores de riesgo asociados con la enfermedad de caries dental en niños. Rev Cubana Estomatol 2003; 40: 23 - 24.

14. Steiner M, Helfenstein U, Marthaler TM. Dental Predictors of highs caries increment in children. J Dent Res 1992; $71: 26-33$.

15. López O, Cerezo M. Puesta a prueba de un Instrumento que predice el riesgo para caries dental, aplicado en adolescentes entre 15 y 19 años, Estrato Socioeconómico 3, del municipio de Manizales, Memorias XVI Encuentro Nacional y IV Internacional de Investigación Odontológica ACFO- Cartagena de Indias 2005; 35.

16. Vale GC, Tabchoury CP, Arthur RA, Del Bel Cury AA, Paes Leme AF, Cury JA. Temporal relationship between sucrose - associated changes in dental biofilm composition and enamel demineralization. Caries Res 2007; 41: 406 - 412.

17. Andreasen PB. Prevention and treatment of dental Caries. Consensus Report. Dan Med Bull 1986; 33: 199 - 202.

18. Rölla G, Holund U, Koch G. Dental Caries: Prevention. En: Koch G, Modeér T, Poulsen S, Rasmussen R. Pedodontics A Clinical Approach. Cap. VI. Editorial Munksgaard, Denmark 1991; $35-40$.

19. Menaker L, Navia JM. Effect of undernutrition during the perinatal period in caries development in the rat: V. Changes in whole saliva volume and protein content. J Dent Res 1974; 53: 592 - 597.

20. Dawes C. An analysis of factors influencing diffusion from dental plaque into a moving film of saliva and the implications for caries. J Dent Res 1989; 68: 1483 -1488 .

21. Finlayson TL, Siefert K, Ismail AI, Sohn W. Psychosocial factors and early childhood caries among low- income Africa American children in Detroit. Community Dent Oral Epidemiol 2007; 35: 439 - 448.

22. González F, Luna L, Martínez N, Solana M. Relación entre caries y factores de riesgo en niños preescolares del Instituto de Bienestar Familiar - Cartagena. Revista de la Academia Colombiana de Odontología Pediátrica 2007; 5: 60 [resumen].

23. Honkala E, Freeman R. Oral hygiene behaviour and periodontal status in European adolescents: an overview. Community Dent Oral Epidemiol 1988; 16: 194 - 198.

24. González F, Alfaro L, Nieto C. Relación de placa bacteriana y caries en niños escolarizados entre 5 y 14 años de la población de la Boquilla - Cartagena. Rev. DUAZARY Universidad del Magdalena 2007; 4: 119-126

25. Samper B, Manjarrez L, Álvarez M, Gómez L, Forero Y, Correa J, Estrada A, De Ossa G. Encuesta Nacional de la situación nutricional en Colombia ENSIN 2005. Instituto Colombiano de Bienestar Familiar. República de Colombia. 
26. Normas Técnicas y Administrativas para la Investigación en Seres Humanos. Resolución 008430 Ministerio de Salud. República de Colombia 1993; 25-26

27. Corchuelo J, Trillos Z. Sensibilidad de un nuevo indicador de placa dental de uso comunitario para el monitoreo de programas dirigidos a grupos del plan de atención básica y del POS. Memorias ACFO 1996; 102 - 109.

28. Ismail AI, Sohn W, Tellez M , Amaya A, Sen A, Hasson H, Pitts NB. The International Caries Detection and Assessment System (ICDAS): and integrated system for measuring dental Caries. Community Dent Oral Epidemiol 2006; 34: $1-9$.

29. Luna, L. Solana, M. Martínez, N. Programa de intervención oral en niños del ICBF de Cartagena [Trabajo de Grado]. Cartagena: Universidad de Cartagena; 2003.

30. González MC, Ruiz JA, Martignon E. Comparison of the def index with Nyvad caries diagnostic criteria in 3 and 4 years old Colombia children. Pediatr Dent 2003; 25: $132-136$.

31. Peres MA, Rosário M, de Oliveira Latorre Mdo R, Sheihan A, Peres KG, Barros FC, Hernández PG, Nuñez AM, Romano $A R$, Victoria CG. Social and biological early life influences on severity of dental caries in children aged 6 years. Community Dent Oral Epidemiol 2005; 33: 53 - 63.

32. Cook S, Martínez A, Dean J, Weddel J, Sanders B, Eggertsson H, Ofner S, Yoder K. Dental Caries experience and association to risk indicators of remote rural populations. Int J Paediatric Dent 2008; 18: 275 - 283.
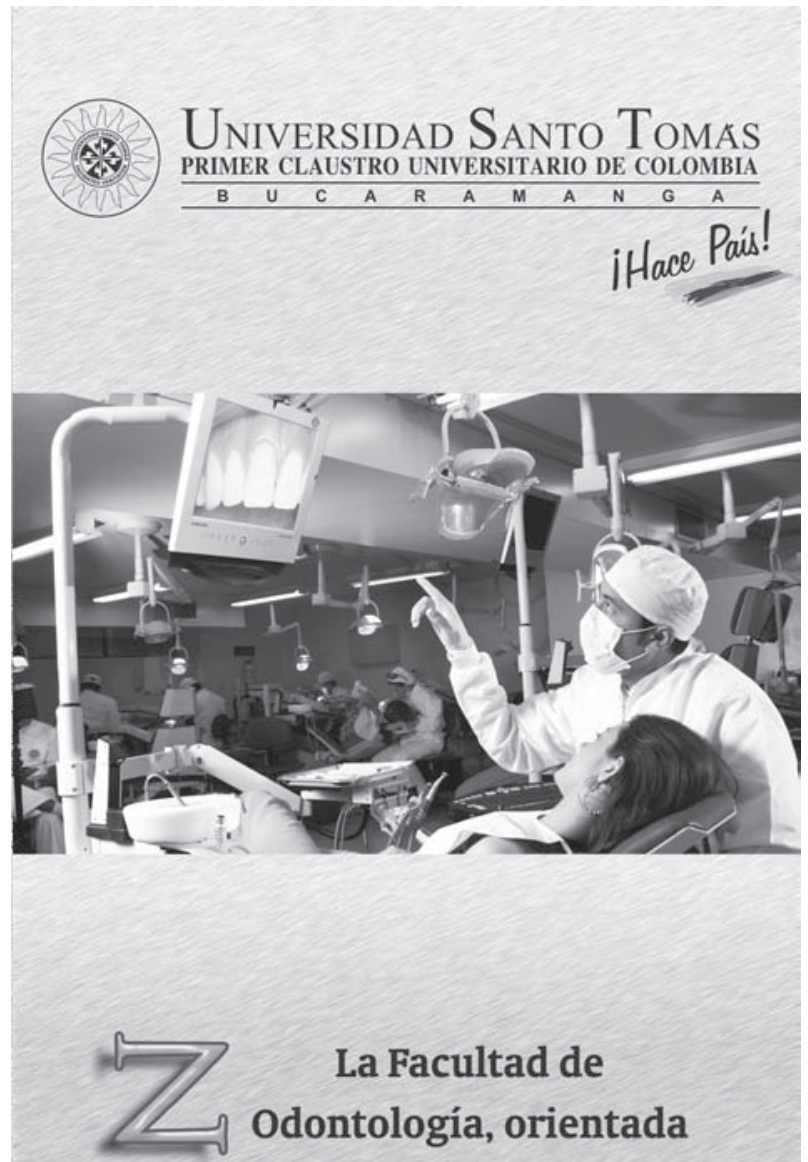

La Facultad de

Odontología, orientada por el pensamiento de Santo Tomás, pretende

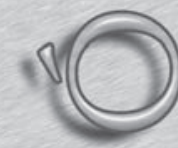
formar Odontólogos integrales $\mathrm{y} / 0$ especialistas a través de parámetros

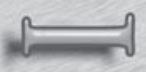

humanísticos, éticos, científicos, biotecnológicos, investigativos $\mathrm{y}$

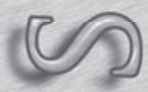

sociales, como recurso

humano capaz de

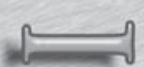
intervenir con éxito en el proceso dinámico de la salud y la enfermedad, en el individuo, la familia y la comunidad. 